TRANSACTIONS OF THE

AMERICAN MATHEMATICAL SOCIETY

Volume 358, Number 10, October 2006, Pages 4441-4465

S 0002-9947(06)03885-2

Article electronically published on February 20, 2006

\title{
TRANSPLANTATION AND MULTIPLIER THEOREMS FOR FOURIER-BESSEL EXPANSIONS
}

\author{
ÓSCAR CIAURRI AND KRZYSZTOF STEMPAK
}

\begin{abstract}
Proved are weighted transplantation inequalities for Fourier-Bessel expansions. These extend known results on this subject by considering the largest possible range of parameters, allowing more weights and admitting a shift. The results are then used to produce a fairly general multiplier theorem with power weights for considered expansions. Also fractional integral results and conjugate function norm inequalities for these expansions are proved.
\end{abstract}

\section{INTRODUCTION AND STATEMENT OF RESULTS}

Given $\nu>-1$, let $\lambda_{n, \nu}, n=1,2, \ldots$, denote the sequence of positive zeros of the Bessel function $J_{\nu}(z)$. Then the functions

$$
\psi_{n}^{\nu}(x)=d_{n, \nu}\left(\lambda_{n, \nu} x\right)^{1 / 2} J_{\nu}\left(\lambda_{n, \nu} x\right), \quad d_{n, \nu}=\sqrt{2}\left|\lambda_{n, \nu}^{1 / 2} J_{\nu+1}\left(\lambda_{n, \nu}\right)\right|^{-1},
$$

$n=1,2, \ldots$, form a complete orthonormal system in $L^{2}((0,1), d x)$. In particular,

$$
\psi_{n}^{-1 / 2}(x)=\sqrt{2} \cos (\pi(n-1 / 2) x), \quad \psi_{n}^{1 / 2}(x)=\sqrt{2} \sin (\pi n x),
$$

for $n=1,2, \ldots$ Given a function $f$ on $(0,1)$, we associate to it its Fourier-Bessel series

$$
f(x) \sim \sum_{1}^{\infty} c_{n}^{\nu}(f) \psi_{n}^{\nu}(x), \quad c_{n}^{\nu}(f)=\int_{0}^{1} f(x) \psi_{n}^{\nu}(x) d x,
$$

provided that the coefficients exist. A comprehensive study of Fourier-Bessel expansions is contained in Chapter XVII of Watson's monograph [9].

The main goal of this paper is to prove a general transplantation theorem for Fourier-Bessel expansions. Transplantation theorems for both discrete and continuous orthogonal transforms were discussed by a number of authors. Muckenhoupt [5. Theorem (1.6)] proved a transplantation result for the orthonormalized Jacobi polynomials. His theorem included the full range of admissible parameters, greatly enlarged the set of applicable weights, allowed a shift in the order parameter and, finally, admitted moment conditions.

Our result is heavily inspired by that of Muckenhoupt: we follow his concept of a "general transplantation theorem" (except the fact that we do not include moment

Received by the editors February 16, 2004 and, in revised form, August 24, 2004.

2000 Mathematics Subject Classification. Primary 42C10; Secondary 44A20.

Key words and phrases. Fourier-Bessel expansions, transplantation, multipliers, weighted norm inequalities, fractional integrals.

The research of the first author was supported by grant BFM2003-06335-603-03 of the DGI.

The research of the second author was supported by KBN grant \#2 P03A 02825.

(C)2006 American Mathematical Society Reverts to public domain 28 years from publication 
conditions) and, proving the result, we use a similar procedure based on showing appropriate bounds, or asymptotics, of the corresponding transplantation kernel.

Given $1 \leq p<\infty$ and a real number $a$, by $L^{p, a}=L^{p, a}(0,1)$ we denote the weighted Lebesgue space of all (equivalence classes of) measurable functions $g$ on $(0,1)$ for which the quantity

$$
\|g\|_{p, a}=\left(\int_{0}^{1}|g(x)|^{p} x^{a} d x\right)^{1 / p}
$$

is finite. For $a=0$ we simplify the notation writing $\|g\|_{p}$ and $L^{p}(0,1)$.

Theorem 1.1. Let $\mu, \nu>-1,1<p<\infty, m$ be an integer, and

$$
-p(\nu+1 / 2)-1<a<p(\mu+3 / 2)-1 .
$$

Then the series $T_{r} f(x)=\sum_{n=1}^{\infty} r^{n} c_{n}^{\mu}(f) \psi_{n+m}^{\nu}(x)$ converges for every $x \in(0,1)$, $f \in L^{p, a}$ and $0<r<1$, and

$$
\left\|T_{r} f(x)\right\|_{p, a} \leq C\|f\|_{p, a},
$$

with $C$ independent of $f \in L^{p, a}$ and $0<r<1$. Moreover, there exists a (unique) bounded operator $T$ on $L^{p, a}$ such that for every $f \in L^{p, a}$

$$
\left\|T_{r} f-T f\right\|_{p, a} \rightarrow 0, \quad r \rightarrow 1^{-} .
$$

If, in addition,

$$
a<p(\nu+3 / 2)-1,
$$

then

$$
c_{n}^{\nu}(T f)= \begin{cases}0, & 1 \leq n \leq m, \\ c_{n-m}^{\mu}(f), & \max \{1, m+1\} \leq n<\infty .\end{cases}
$$

Here and later on we assume $\psi_{k}^{\nu}=0$ if $k \leq 0$. Note that the assumption $a<p(\mu+3 / 2)-1$ guarantees the existence of the coefficients $c_{n}^{\mu}(f)$ whenever $f \in L^{p, a}$, while the hypothesis $-p(\nu+1 / 2)-1<a$ implies that $\psi_{n+m}^{\nu}$ belong to $L^{p, a}$ for all $n=1,2, \ldots$ (Hölder's inequality and the estimate (2.8) are used). Therefore, (1.1) should be seen as a natural (minimal) assumption. In the same way, (1.4) ensures the existence of the coefficients $c_{n}^{\nu}(T f)$ for any $f \in L^{p, a}$.

Corollary 1.2. Let $\mu, \nu, p, m$ and a satisfy the assumptions of Theorem $1.1 \mathrm{ex}$ cept for the assumption (1.4). The operator $T$ that appears in the conclusions of Theorem 1.1 is the unique bounded on $L^{p, a}$ extension of the operator

$$
f \mapsto \sum_{n=1}^{\infty} c_{n}^{\mu}(f) \psi_{n+m}^{\nu}(x),
$$

initially defined on $C_{c}^{\infty}(0,1)$. If, in addition, $-p(\mu+1 / 2)-1<a$, then $T$ is also the unique bounded on $L^{p, a}$ extension of the operator

$$
\psi_{n}^{\mu} \mapsto \psi_{n+m}^{\nu}, \quad n=1,2, \ldots,
$$

initially defined on the subspace spanned by $\left\{\psi_{n}^{\mu}: n=1,2, \ldots\right\}$.

The choices of $\mu=-1 / 2$ and $\nu=1 / 2$ as well as $\mu=1 / 2$ and $\nu=-1 / 2$ in Corollary 1.2 (together with taking $m=0$ ) show that for $1<p<\infty$ and $-1<a<p-1$ we have

$$
C^{-1}\left\|\sum a_{n} \sin (\pi n x)\right\|_{p, a} \leq\left\|\sum a_{n} \cos (\pi(n-1 / 2) x)\right\|_{p, a} \leq C\left\|\sum a_{n} \sin (\pi n x)\right\|_{p, a},
$$


with $C$ independent of sequences $\left\{a_{n}\right\}_{n=1}^{\infty}$ such that $a_{n}=0$ for all but a finite number of $n$ 's. This may be seen as a variation of M. Riesz's conjugacy theorem.

Corollary 1.2 specified to the case $m=0$ gives the Fourier-Bessel transplantation inequality

$$
\left\|\sum_{n=1}^{\infty} c_{n}^{\mu}(f) \psi_{n}^{\nu}(x)\right\|_{p, a} \leq C\|f\|_{p, a}, \quad f \in C_{c}^{\infty}(0,1) .
$$

Therefore, by using [1, Theorem 2.4], the above produces the Hankel transform transplantation inequality

$$
\left\|\left(\mathcal{H}_{\nu} \circ \mathcal{H}_{\mu}\right) f\right\|_{p, a} \leq C\|f\|_{p, a}, \quad f \in C_{c}^{\infty}(0, \infty),
$$

with the range of $a$ given by (1.1), where $\mathcal{H}_{\nu}$ is the Hankel transform of order $\nu$,

$$
\mathcal{H}_{\nu} f(x)=\int_{0}^{\infty}(x y)^{1 / 2} J_{\nu}(x y) f(y) d y, \quad x>0
$$

for any appropriate function $f$ on $(0, \infty)$. This approach seems to be, in our opinion, the most straightforward for obtaining an optimal Hankel transform transplantation inequality (with power weights) by means of a transference argument; cf. also [8] .

Specified to the case of $m= \pm 1$, Corollary 1.2 also shows that the forward and backward shift operators

$$
\psi_{n}^{\nu} \mapsto \psi_{n-1}^{\nu}, \quad \psi_{n}^{\nu} \mapsto \psi_{n+1}^{\nu},
$$

uniquely extend to operators bounded on $L^{p, a}$, provided that $1<p<\infty$ and $-p(\nu+1 / 2)-1<a<p(\nu+3 / 2)-1$. These operators may by seen as conjugate transforms in the setting of expansions with respect to the system $\left\{\psi_{n}^{\nu}\right\}$.

Theorem 1.3. Let $\mu, \nu>-1,1<p<\infty, 0<s<\min \{1 / p, \mu+\nu+2\}$, $m$ be an integer, $1 / q=1 / p-s$ and

$$
-(\nu+1 / 2)-1 / q<a<\mu+3 / 2-1 / p
$$

Then the series $T_{r} f(x)=\sum_{n=1}^{\infty} r^{n} n^{-s} c_{n}^{\mu}(f) \psi_{n+m}^{\nu}(x)$ converges for every $x \in(0,1)$, $f \in L^{p, a p}$ and $0<r<1$, and

$$
\left\|T_{r} f\right\|_{q, a q} \leq C\|f\|_{p, a p}
$$

with $C$ independent of $f \in L^{p, a p}$ and $0<r<1$. Moreover, there exists a (unique) bounded operator $T$ from $L^{p, a p}$ into $L^{q, a q}$ such that for every $f \in L^{p, a p}$

$$
\left\|T_{r} f-T f\right\|_{q, a q} \rightarrow 0, \quad r \rightarrow 1^{-} .
$$

If, in addition,

$$
a<\nu+3 / 2-1 / q
$$

then

$$
c_{n}^{\nu}(T f)= \begin{cases}0, & 1 \leq n \leq m, \\ (n-m)^{-s} c_{n-m}^{\mu}(f), & \max \{1, m+1\} \leq n<\infty .\end{cases}
$$

Corollary 1.4. Let $\mu, \nu, p, q, m, s$ and a satisfy the assumptions of Theorem 1.3 except for the assumption (1.9). The operator $T$ that appears in the conclusions of that theorem is the unique bounded from $L^{p, a p}$ into $L^{q, a q}$ extension of the operator

$$
f \mapsto \sum_{n=1}^{\infty} n^{-s} c_{n}^{\mu}(f) \psi_{n+m}^{\nu}(x)
$$


initially defined on $C_{c}^{\infty}(0,1)$. If, in addition, $-(\mu+1 / 2)-1 / p<a$, then $T$ is also the unique bounded from $L^{p, a p}$ into $L^{q, a q}$ extension of the operator

$$
\psi_{n}^{\mu} \mapsto \psi_{n+m}^{\nu}, \quad n=1,2, \ldots,
$$

initially defined on the subspace spanned by $\left\{\psi_{n}^{\mu}: n=1,2, \ldots\right\}$.

Of particular interest is the case of $m=0$ and $\mu=\nu$. Then Corollary 1.4 says that the fractional integral operator

$$
I_{s} f(x)=\sum_{n=1}^{\infty} n^{-s} c_{n}^{\mu}(f) \psi_{n}^{\mu}(x),
$$

initially defined on $C_{c}^{\infty}(0,1)$, extends to a bounded operator from $L^{p, a p}$ into $L^{q, a q}$, provided the relevant assumptions are satisfied.

For a multiplier theorem we now state that we use the same classes of multiplier sequences, $M(t, \lambda)$, as those in [5, p.51] (apart from the fact that our sequences are indexed by the positive integers). For a positive integer $\lambda$ and $1 \leq t \leq \infty$ they are the sequences $\left\{m_{n}\right\}_{n=1}^{\infty}$ satisfying the Hörmander-type condition

$$
B(m, t, \lambda)=\left\|m_{n}\right\|_{\infty}+\sup _{k} 2^{k(\lambda-1 / t)}\left(\sum_{n=2^{k}}^{2^{k+1}}\left|\Delta^{\lambda} m_{n}\right|^{t}\right)^{1 / t}<\infty,
$$

where $\Delta^{\lambda}=\Delta\left(\Delta^{\lambda-1}\right)$ and $\Delta=\Delta^{1}$ denotes the forward difference operator, $\Delta m_{n}=$ $m_{n+1}-m_{n}$. For the definition of $M(t, \lambda)$ with an arbitrary positive $\lambda$ and more information on these class of multipliers, we refer the reader to $[5]$.

Theorem 1.5. Let $\nu>-1,1<p<\infty, 1 \leq t \leq \infty, \lambda>\max \{1 / t,|1 / p-1 / 2|\}$ or $\lambda=t=1, m \in M(t, \lambda),|a|<p \lambda$ and

$$
\begin{aligned}
& \max \{-1,-1-p(\nu+1 / 2),-1+p(-\lambda+1 / 2)\}<a \\
& \quad<\min \{2 p-1,-1+p(\nu+3 / 2),-1+p(\lambda+1 / 2),-1+p(\lambda+1-1 / t)\} .
\end{aligned}
$$

Then

$$
\left\|\sum_{n=1}^{\infty} m_{n} d_{n} \psi_{n}^{\nu}\right\|_{p, a} \leq C B(m, t, \lambda)\left\|\sum_{n=1}^{\infty} d_{n} \psi_{n}^{\nu}\right\|_{p, a}
$$

with $C$ independent of $m$ and $d$, and any sequence $\left\{d_{n}\right\}_{n=1}^{\infty}$ such that $d_{n}=0$ for all but a finite number of n's. Moreover, the operator initially defined on the span of $\left\{\psi_{n}^{\nu}: n=1,2, \ldots\right\}$ by $\psi_{n}^{\nu} \mapsto m_{n} \psi_{n}^{\nu}$ uniquely extends to a bounded operator on $L^{p, a}$.

A transplantation theorem for Fourier-Bessel expansions is contained in Theorem A of Gilbert's paper [2]. This theorem states a general result of transplantation type for operators with kernels satisfying a number of "natural" conditions. The Fourier-Bessel expansions fit into that frame, and, moreover, a proper modification of Gilbert's argument leads to a more general weighted result with $A_{p}$ weights involved; cf. [3]. 


\section{Preliminaries}

We recall some known facts related to the Fourier-Bessel system (see [1]):

$$
\begin{aligned}
& d_{n, \nu}=\pi^{1 / 2}\left(1+j_{n, \nu}\right), \quad j_{n, \nu}=O\left(n^{-1}\right), \\
& \lambda_{n, \nu}=\pi\left(n+D_{\nu}+c_{n, \nu}\right), \quad c_{n, \nu}=O\left(n^{-1}\right),
\end{aligned}
$$

$D_{\nu}=(2 \nu-1) / 4$. In fact, the precise asymptotics of the sequence $\lambda_{n, \nu}$ is known (see [9, p. 506]):

$$
\lambda_{n, \nu} \sim \pi\left(n+D_{\nu}+\sum_{k=0}^{\infty} \frac{R_{\nu, k}}{\left(n+D_{\nu}\right)^{2 k+1}}\right),
$$

which means that there is a sequence of coefficients $\left\{R_{\nu, k}\right\}_{k=0}^{\infty}$, such that for any given positive integer $p$,

$$
\lambda_{n, \nu}=\pi\left(n+D_{\nu}+\sum_{k=0}^{p-1} \frac{R_{\nu, k}}{\left(n+D_{\nu}\right)^{2 k+1}}\right)+O\left(n^{-(2 p+1)}\right) .
$$

It is therefore clear that given a nonnegative integer $\ell$ one has

$$
\lambda_{n, \nu}=\sum_{k=-1}^{\ell} \frac{S_{\nu, k}}{n^{k}}+O\left(n^{-\ell-1}\right) .
$$

We will use the well-known estimates for the Bessel functions: for $\nu>-1$

$$
J_{\nu}(z)=O\left(z^{\nu}\right), \quad z \rightarrow 0^{+},
$$

and, given a nonnegative integer $M$,

$$
\sqrt{z} J_{\nu}(z)=\sum_{j=0}^{M}\left(\frac{A_{\nu, j}}{z^{j}} \sin z+\frac{B_{\nu, j}}{z^{j}} \cos z\right)+H_{M}(z), \quad z \rightarrow \infty,
$$

where $\left|H_{M}(z)\right| \leq C z^{-(M+1)}$ (see 4, p. 122], for example). A straightforward calculation shows that using (2.4) and (2.6) produces

$$
d_{n, \nu}=\sum_{k=0}^{\ell} \frac{Q_{\nu, k}}{n^{k}}+O\left(n^{-\ell-1}\right)
$$

for any given nonnegative integer $\ell$.

On the other hand it follows from (2.5), (2.6) and (2.1) that

$$
\left|\psi_{n}^{\nu}(x)\right| \leq C \begin{cases}(n x)^{\nu+1 / 2}, & 0<x<n^{-1} \\ 1, & n^{-1}<x<1 .\end{cases}
$$

Lemma 2.1. Let $a<p(\mu+3 / 2)-1$. Then, for any $f \in L^{p, a}$,

$$
c_{n}^{\mu}(f)=O\left(n^{\rho}\right), \quad n=1,2, \ldots,
$$

for a $\rho=\rho(f, \mu, p, a)$. If $-p(\nu+1 / 2)-1<a$, then

$$
\left\|\psi_{n}^{\nu}\right\|_{p, a}=O\left(n^{\tau}\right), \quad n=1,2, \ldots,
$$

for $a \tau=\tau(\nu, p, a)$. 
Proof. We write

$$
\begin{aligned}
& \left|c_{n}^{\mu}(f)\right| \leq C\left(n^{\mu+1 / 2} \int_{0}^{1 / n}|f(x)| x^{\mu+1 / 2} d x+\int_{1 / n}^{1}|f(x)| d x\right) \\
& \leq C\|f\|_{p, a}\left(n^{\mu+1 / 2}\left(\int_{0}^{1 / n} x^{(\mu+1 / 2-a / p) p^{\prime}} d x\right)^{1 / p^{\prime}}+\left(\int_{1 / n}^{1} x^{-a p^{\prime} / p} d x\right)^{1 / p^{\prime}}\right)
\end{aligned}
$$

and (2.9) follows. The assumption on $a$ was used to ensure the convergence of the integral over $(0,1 / n)$. A similar calculation shows (2.10).

Lemma 2.2. For any positive integer $k$ and $h \in C_{c}^{\infty}(0,1)$,

$$
\left|c_{n}^{\nu}(h)\right| \leq C_{k, h} n^{-k}, \quad n=1,2, \ldots
$$

Proof. The functions $\psi_{n}^{\nu}, n=1,2, \ldots$, are eigenfunctions of the differential operator (symmetric in $L^{2}(0,1)$ )

$$
L_{\nu}=\frac{d^{2}}{d x^{2}}+\frac{1 / 4-\nu^{2}}{x^{2}} .
$$

More precisely, $L_{\nu} \psi_{n}^{\nu}(x)=-\lambda_{n, \nu}^{2} \psi_{n}^{\nu}(x)$. Thus $\left\langle h, \psi_{n}^{\nu}\right\rangle=-\lambda_{n, \nu}^{-2}\left\langle L_{\nu} h, \psi_{n}^{\nu}\right\rangle$, and repeating this procedure and using Schwarz's inequality produces (2.11).

Lemma 2.3. Let $1<p<\infty$ and $-p(\nu+1 / 2)-1<a$. Then the subspace spanned by $\left\{\psi_{n}^{\nu}: n=1,2, \ldots\right\}$ is dense in $L^{p, a}$.

Proof. It is sufficient to approximate functions from $C_{c}^{\infty}(0,1)$ by linear combinations of $\psi_{n}^{\nu}$ 's. Fix $f \in C_{c}^{\infty}(0,1)$. We will show that a subsequence of the partial sums,

$$
S_{N} f=\sum_{n=1}^{N} c_{n}^{\nu}(f) \psi_{n}^{\nu}
$$

approaches $f$ in $L^{p, a}$. Indeed, $f=\lim _{N \rightarrow \infty} S_{N} f$ in the $L^{2}(0,1)$ sense. Therefore, we can find a subsequence $N(1)<N(2)<\ldots$ such that $f(x)=\lim _{m \rightarrow \infty} S_{N(m)} f(x)$, a.e. in $(0,1)$. Using (2.11) now gives

$$
\left|S_{N} f(x)\right| \leq C \sum_{1 \leq n \leq N} n^{-k}\left|\psi_{n}^{\nu}(x)\right|,
$$

and then, for $1<p<\infty$, Hölder's inequality produces

$$
\left|S_{N} f(x)\right|^{p} \leq C\left(\sum_{n=1}^{\infty} n^{-k}\right)^{p / p^{\prime}} \sum_{n=1}^{\infty} n^{-k}\left|\psi_{n}^{\nu}(x)\right|^{p} .
$$

Therefore, for $1<p<\infty$, choosing in Lemma $2.2 k$ large enough shows that the sequence of functions $\left|S_{N} f(x)\right|^{p}, N=1,2, \ldots$, is majorized, up to a constant factor, by the function $\sum_{n} n^{-k}\left|\psi_{n}^{\nu}(x)\right|^{p}$ which is in $L^{1, a}$. Therefore, using the dominated convergence theorem, $S_{N(m)} f \rightarrow f$ in $L^{p, a}$.

We will frequently use, without further mention, the fact that

$$
\sum_{n=1}^{N} n^{\rho}= \begin{cases}O\left(N^{\rho+1}\right), & \text { for } \rho>-1, \\ O(\log N), & \text { for } \rho=-1,\end{cases}
$$


and

$$
\sum_{n=N}^{\infty} n^{\rho}=O\left(N^{\rho+1}\right), \quad \rho<-1 .
$$

Also, we will use the summation by parts formula

$$
\sum_{n=N}^{\infty} a_{n} \Delta b_{n}=-a_{N} b_{N}-\sum_{n=N}^{\infty} b_{n+1} \Delta a_{n}
$$

where $\Delta a_{n}=a_{n+1}-a_{n}$. By $f * g$ we will denote the convolution,

$$
g * h(x)=\int_{-\pi}^{\pi} g(y) h(x-y) d y,
$$

of two $2 \pi$-periodic functions $g$ and $h$ on $(-\pi, \pi)$.

Finally, we recall the following two forms of Hardy's inequality: if $a<-1$ and $1 \leq p<\infty$, then

$$
\int_{0}^{1}\left|\int_{0}^{x} f(t) d t\right|^{p} x^{a} d x \leq C \int_{0}^{1}|f(x)|^{p} x^{a+p} d x
$$

if $a>-1$ and $1 \leq p<\infty$, then

$$
\int_{0}^{1}\left|\int_{x}^{1} f(t) d t\right|^{p} x^{a} d x \leq C \int_{0}^{1}|f(x)|^{p} x^{a+p} d x .
$$

As usual, $p^{\prime}$ will denote the conjugate value to $p, 1<p<\infty$, and [ ] will mean the greatest integer function; $C_{c}^{\infty}(0,1)$ will mean the space of $C^{\infty}$-functions on $(0,1)$ with compact support. Constants with subscripts will indicate a dependence on these subscripts.

\section{Preparatory lemmas}

In this section we state and prove three technical lemmas. Lemma 3.1 together with Lemma 3.2 serve in proving Lemma 3.3. which is one of key ingredients in the proofs of our main estimates, Proposition 4.3 and Proposition 5.1 .

Let

$$
P_{r}(x)=\frac{1}{2}+\sum_{n=1}^{\infty} r^{n} \cos (n x)=\frac{1-r^{2}}{2\left(1-2 r \cos x+r^{2}\right)}
$$

be the usual Poisson kernel and

$$
Q_{r}(x)=\sum_{n=1}^{\infty} r^{n} \sin (n x)=\frac{r \sin x}{1-2 r \cos x+r^{2}}
$$

be the conjugate Poisson kernel. The following identities are obvious:

$$
\begin{aligned}
& (-1)^{k} \frac{d^{2 k} Q_{r}(x)}{d x^{2 k}}=\sum_{n=1}^{\infty} r^{n} n^{2 k} \sin (n x), \quad(-1)^{k} \frac{d^{2 k+1} Q_{r}(x)}{d x^{2 k+1}}=\sum_{n=1}^{\infty} r^{n} n^{2 k+1} \cos (n x), \\
& (-1)^{k} \frac{d^{2 k} P_{r}(x)}{d x^{2 k}}=\sum_{n=1}^{\infty} r^{n} n^{2 k} \cos (n x), \quad(-1)^{k+1} \frac{d^{2 k+1} P_{r}(x)}{d x^{2 k+1}}=\sum_{n=1}^{\infty} r^{n} n^{2 k+1} \sin (n x)
\end{aligned}
$$

(to be precise in the third identity we assume $k \geq 1$; otherwise, for $k=0$, we must include the summand $1 / 2$ on the right). 
Lemma 3.1. For $n=0,1, \ldots, 0<r<1$ and $0<|x|<3 \pi / 2$, we have

$$
\left|\frac{d^{n} P_{r}(x)}{d x^{n}}\right| \leq C_{n}|x|^{-(n+1)}, \quad\left|\frac{d^{n} Q_{r}(x)}{d x^{n}}\right| \leq C_{n}|x|^{-(n+1)} .
$$

Proof. For either $P_{r}$ or $Q_{r}$ the $n$th derivative has the form

$$
\frac{S_{n}(r, x)}{\left((1-r)^{2}+4 r \sin ^{2} \frac{x}{2}\right)^{n+1}},
$$

where

$$
S_{n+1}(r, x)=\left((1-r)^{2}+4 r \sin ^{2} \frac{x}{2}\right) \frac{d S_{n}(r, x)}{d x}-(n+1) 4 r \sin \frac{x}{2} \cos \frac{x}{2} S_{n}(r, x)
$$

and $S_{0}(r, x)=\frac{1}{2}\left(1-r^{2}\right)$ in the case of $P_{r}$ or $S_{0}(r, x)=r \sin x$ in the case of $Q_{r}$. It follows inductively that $S_{n}(r, x)$ can be written as the sum of terms of the form $f(r, x)(1-r)^{j} \sin ^{k} \frac{x}{2}$, where $f(r, x)$ is a bounded function and $j+k \geq n+1$. From this $\left|S_{n}(r, x)\right| \leq C\left((1-r)^{n+1}+\left|\sin \frac{x}{2}\right|^{n+1}\right)$. The result follows immediately by separately considering $1-r \leq|x|<3 \pi / 2$ and $0<|x|<1-r$.

Lemma 3.2. Let $0 \leq \beta<1$ and $f$ be a $2 \pi$-periodic function such that $|f(x)| \leq$ $C|x|^{-\beta}$ for $0<|x| \leq \pi$. Then, the estimate

$$
\left|P_{r} * f(x)\right| \leq C|x|^{-\beta}
$$

holds, with $C=C_{\beta}$ independent of $0<r<1$ and $0<|x|<3 \pi / 2$.

Proof. It is sufficient to check that $\left|P_{r} * f(x)\right| \leq C|x|^{-\beta}$ for $0<|x|<\pi / 2$ and $\left|P_{r} * f(x)\right| \leq C$ for $\pi / 2 \leq|x| \leq 3 \pi / 2$ with constants independent of $r$ and $x$. Since the periodicity of $f$ allows the hypothesized estimate $|f(x)| \leq C|x|^{-\beta}$ to hold for $0<|x| \leq 3 \pi / 2$, the proof of the first bound reduces to showing that

$$
\int_{0}^{\pi} P_{r}(y)|x \pm y|^{-\beta} d y \leq C x^{-\beta}, \quad 0<x<\pi / 2 .
$$

To show (3.1) we split the integration region $(0, \pi)$ into two parts, $A=(0, x / 2) \cup$ $(2 x, \pi)$ and $B=(x / 2,2 x)$. The fact that $\int_{-\pi}^{\pi} P_{r}(t) d t=\pi$ easily gives

$$
\int_{A} P_{r}(y)|x \pm y|^{-\beta} d y \leq C x^{-\beta} .
$$

On the other hand, by Lemma $3.1 P_{r}(t) \leq C t^{-1}$ for $0<t<\pi$, hence,

$$
\int_{B} P_{r}(y)|x \pm y|^{-\beta} d y \leq C x^{-1} \int_{x / 2}^{2 x}|x \pm y|^{-\beta} d y \leq C x^{-\beta} .
$$

The proof of the second bound reduces to showing that

$$
\int_{0}^{\pi} y^{-\beta} P_{r}(y \pm x) d y \leq C, \quad \pi / 2 \leq x \leq 3 \pi / 2 .
$$

The estimate follows since

$$
\int_{0}^{\pi} y^{-\beta} P_{r}(y \pm x) d y \leq A_{ \pm} \int_{0}^{\pi / 4} y^{-\beta} d y+(\pi / 4)^{-\beta} \int_{\pi / 4}^{\pi} P_{r}(x \pm y) d y,
$$

where

$$
A_{-}=\sup _{0<r<1} \sup _{\pi / 4 \leq t \leq 3 \pi / 2} P_{r}(t), \quad A_{+}=\sup _{0<r<1} \sup _{\pi / 2 \leq t \leq 7 \pi / 4} P_{r}(t) .
$$


Lemma 3.3. Given $\gamma>0$ the estimates

$$
\left|\sum_{n=1}^{\infty} r^{n} n^{\gamma-1}\left\{\begin{array}{c}
\sin \\
\cos
\end{array}\right\}(n x)\right| \leq C_{\gamma}|x|^{-\gamma}
$$

hold with a constant $C_{\gamma}$ independent of $0<r<1$ and $0<|x|<3 \pi / 2$.

Proof. For $\gamma=m, m=1,2, \ldots$, the result is contained in Lemma 3.1 To prove (3.2) for other values of $\gamma$ we proceed by induction. The following estimate is stated in [10, (13), p. 70, Vol. I]: for any $0<\gamma<1$ and $0<|x|<3 \pi / 2$

$$
\left|\sum_{n=1}^{\infty} n^{\gamma-1}\left\{\begin{array}{l}
\sin \\
\cos
\end{array}\right\}(n x)\right| \leq C_{\gamma}|x|^{-\gamma} .
$$

This, together with Lemma 3.2 shows (3.2) for $0<\gamma<1$.

Suppose now that $m<\gamma<m+1$ with $m \geq 1$. To simplify the notation write

$$
S_{\gamma}(x)=S_{\gamma}(r, x)=\sum_{n=1}^{\infty} r^{n} n^{\gamma-1} \sin (n x), \quad C_{\gamma}(x)=C_{\gamma}(r, x)=\sum_{n=1}^{\infty} r^{n} n^{\gamma-1} \cos (n x) .
$$

We will show that

$$
\begin{aligned}
S_{\gamma}(x)=S_{m}(x)+\sum_{s=1}^{m} \sum_{p=s}^{m} a_{s, p, \gamma} & \left(S_{s}(x) C_{\gamma-p}(x)+C_{s}(x) S_{\gamma-p}(x)\right) \\
& +\sum_{s=1}^{m}\left(S_{s}(x) A_{s, \gamma}(r, x)+C_{s}(x) B_{s, \gamma}(r, x)\right),
\end{aligned}
$$

where $a_{s, p, \gamma}$ are constants and $A_{s, \gamma}(r, x), B_{s, \gamma}(r, x)$ are bounded functions. An analogous formula holds for $C_{\gamma}$ (to be precise, on the right of (3.3) $, S_{m}, S_{s}, C_{s}$ and the second plus sign have to be replaced by $C_{m}, C_{s}, S_{s}$ and the minus sign), and the proof of it proceeds analogously to that of (3.3) by using the identity for $\cos (a+b)$ in place of a similar one for $\sin (a+b)$. These two identities, together with the induction hypothesis and the estimates (3.2) known to hold for positive integer values of $\gamma$, show (3.2) for $m<\gamma<m+1$.

Applying the summation by parts formula to the series defining $S_{\gamma}(x)$ with $a_{n}=n^{\gamma-m}$ and $b_{n}=-\sum_{k=n}^{\infty} r^{k} k^{m-1} \sin (k x)$ gives

$$
S_{\gamma}(x)=S_{m}(x)+\sum_{n=1}^{\infty}\left((n+1)^{\gamma-m}-n^{\gamma-m}\right) \sum_{k=n+1}^{\infty} r^{k} k^{m-1} \sin (k x) .
$$

The last sum, after renumeration and using the formula for $\sin (a+b)$, equals

$$
r^{n} \sum_{s=1}^{m}\left(\begin{array}{c}
m-1 \\
s-1
\end{array}\right) n^{m-s} \cos (n x) S_{s}(x)+r^{n} \sum_{s=1}^{m}\left(\begin{array}{c}
m-1 \\
s-1
\end{array}\right) n^{m-s} \sin (n x) C_{s}(x),
$$

hence

$$
\begin{aligned}
S_{\gamma}(x)=S_{m}(x) & +\sum_{s=1}^{m}\left(\begin{array}{c}
m-1 \\
s-1
\end{array}\right) S_{s}(x) \sum_{n=1}^{\infty} r^{n}\left((n+1)^{\gamma-m}-n^{\gamma-m}\right) n^{m-s} \cos (n x) \\
& +\sum_{s=1}^{m}\left(\begin{array}{c}
m-1 \\
s-1
\end{array}\right) C_{s}(x) \sum_{n=1}^{\infty} r^{n}\left((n+1)^{\gamma-m}-n^{\gamma-m}\right) n^{m-s} \sin (n x) .
\end{aligned}
$$


For each $s, s=1, \ldots, m$,

$$
\left((n+1)^{\gamma-m}-n^{\gamma-m}\right) n^{m-s}=n^{\gamma-s} \sum_{k=1}^{m-s+1}\left(\begin{array}{c}
\gamma-m \\
k
\end{array}\right) \frac{1}{n^{k}}+p_{n},
$$

where $p_{n}=p_{n}(s, \gamma)$ and $\left|p_{n}\right| \leq C_{s, \gamma} n^{\gamma-m-2}$. Therefore,

$$
\begin{array}{r}
S_{\gamma}(x)=S_{m}(x)+\sum_{s=1}^{m} \sum_{p=s}^{m}\left(\begin{array}{c}
m-1 \\
s-1
\end{array}\right)\left(\begin{array}{c}
\gamma-m \\
p-s+1
\end{array}\right)\left(S_{s}(x) C_{\gamma-p}(x)+C_{s}(x) S_{\gamma-p}(x)\right) \\
+\sum_{s=1}^{m}\left(S_{s}(x)\left(\begin{array}{c}
m-1 \\
s-1
\end{array}\right) \sum_{n=1}^{\infty} r^{n} p_{n} \cos (n x)+C_{s}(x)\left(\begin{array}{c}
m-1 \\
s-1
\end{array}\right) \sum_{n=1}^{\infty} r^{n} p_{n} \sin (n x)\right),
\end{array}
$$

and (3.3) follows.

It is, perhaps, interesting to note that for $\gamma=0$ Lemma 3.3 remains valid for the sine series but fails for the cosine series. This is because, in the sine case,

$$
\sum_{n=1}^{\infty} \frac{1}{n} \sin (n x)=\frac{\pi-x}{2}, \quad 0<x<2 \pi,
$$

and the required bound follows by applying Lemma 3.2 with $\beta=0$, while, in the cosine case,

$$
\sum_{n=1}^{\infty} \frac{1}{n} \cos (n x)=-\ln \left(2 \sin \frac{x}{2}\right), \quad 0<x<2 \pi .
$$

\section{Estimates of the TRANSPLANTATION KERNEL FOR NONCOMPARABLE VALUES OF $x$ AND $y$}

In this section we obtain estimates of the transplantation kernel

$$
L_{s, m}(r, x, y)=\sum_{n=1}^{\infty} r^{n} n^{-s} \psi_{n+m}^{\nu}(x) \psi_{n}^{\mu}(y),
$$

outside the diagonal $x=y$ for noncomparable values of $x$ and $y$. Before proceeding to the main result of this section, Proposition 4.3. we first prove some technical results.

In what follows the notation

$$
\left\{\begin{array}{l}
\sin \\
\cos
\end{array}\right\}(\pi n x)\left\{\begin{array}{l}
\sin \\
\cos
\end{array}\right\}(\pi n y)
$$

will mean that all four possible combinations are allowed but, in any given series, the same combination must occur for all $n$.

Lemma 4.1. Let $\mu, \nu>-1$ and $m$ be an integer. Then each of the functions

$$
\left\{\begin{array}{c}
\sin \\
\cos
\end{array}\right\}\left(\lambda_{n+m, \nu} x\right) \cos \left(\lambda_{n, \mu} y\right), \quad n=1,2, \ldots,
$$

can be written as the sum of four terms of the form

$$
\left\{\begin{array}{l}
\sin \\
\cos
\end{array}\right\}(\pi n x)\left\{\begin{array}{l}
\sin \\
\cos
\end{array}\right\}(\pi n y) W_{n}(x, y)
$$


where

$$
W_{n}(x, y)=\sum_{i=1}^{4} \varepsilon_{i} u_{i}(x) v_{i}(y) U_{i}(n, x) V_{i}(n, y), \quad 0<x, y<1,
$$

$\varepsilon_{i}$ equals either 1 or $-1, u_{i}(x)$ equals either $\sin \left(\pi\left(m+D_{\nu}\right) x\right)$ or $\cos \left(\pi\left(m+D_{\nu}\right) x\right)$, $v_{i}(y)$ equals either $\sin \left(\pi D_{\mu} y\right)$ or $\cos \left(\pi D_{\mu} y\right), U_{i}(n, x)$ equals either $\sin \left(\pi c_{m+n, \nu} x\right)$ or $\cos \left(\pi c_{m+n, \nu} x\right)$, and $V_{i}(n, y)$ equals either $\sin \left(\pi c_{n, \mu} y\right)$ or $\cos \left(\pi c_{n, \mu} y\right)\left(c_{m+n, \nu}\right.$ and $c_{n, \mu}$ are defined by (2.2) ).

Proof. To prove the lemma with the sine factor, $\sin \left(\lambda_{n+m, \nu} x\right)$ (for the cosine factor, $\cos \left(\lambda_{n+m, \nu} x\right)$, the procedure is similar), we apply the identity

$$
\begin{aligned}
\sin (a+b+ & d) \cos (\alpha+\beta+\delta) \\
= & \sin a \sin \alpha(-\cos b \sin \beta \cos d \cos \delta \\
& +\sin b \sin \beta \sin d \cos \delta-\cos b \cos \beta \cos d \sin \delta+\sin b \cos \beta \sin d \sin \delta) \\
+ & \sin a \cos \alpha(\cos b \cos \beta \cos d \cos \delta \\
& -\cos b \sin \beta \cos d \sin \delta+\sin b \sin \beta \sin d \sin \delta-\sin b \cos \beta \sin d \cos \delta) \\
+ & \cos a \sin \alpha(-\sin b \sin \beta \cos d \cos \delta \\
& -\cos b \sin \beta \sin d \cos \delta-\sin b \cos \beta \cos d \sin \delta-\cos b \cos \beta \sin d \sin \delta) \\
+ & \cos a \cos \alpha(\sin b \cos \beta \cos d \cos \delta \\
& +\cos b \cos \beta \sin d \cos \delta-\sin b \sin \beta \cos d \sin \delta-\cos b \sin \beta \sin d \sin \delta),
\end{aligned}
$$

with $a=\pi n x, \alpha=\pi n y, b=\pi\left(m+D_{\nu}\right) x, \beta=\pi D_{\mu} y, d=\pi c_{n+m, \nu} x$ and $\delta=\pi c_{n, \mu} y$. Then (4.2) follows.

Lemma 4.2. Let $\mu, \nu>-1$, let $m$ be an integer, let $U(n, x)$ equal either $\sin \left(\pi c_{m+n, \nu} x\right)$ or $\cos \left(\pi c_{m+n, \nu} x\right)$, and let $V(n, y)$ equal either $\sin \left(\pi c_{n, \mu} y\right)$ or $\cos \left(\pi c_{n, \mu} y\right)$. Given nonnegative integers $j, \ell$ and a real number $\gamma$, for $n=1,2, \ldots$, we have

$$
d_{n+m, \nu} d_{n, \mu} \lambda_{n+m, \nu}^{-j} \lambda_{n, \mu}^{\gamma} U(n, x) V(n, y)=n^{-j+\gamma} E_{j, \gamma, \ell}(n, x, y),
$$

where

$$
E_{j, \gamma, \ell}(n, x, y)=\sum_{k=0}^{\ell} \frac{A_{k}(x, y)}{n^{k}}+q_{n}(x, y),
$$

$A_{k}(x, y), k=0,1, \ldots, \ell$, is a polynomial of the two variables $x$ and $y$ of degree $k$, and $q_{n}(x, y)$, for $0<x, y<1$, satisfies $\left|q_{n}(x, y)\right| \leq C n^{-\ell-1}\left(q_{n}\right.$, as well as the coefficients of $A_{k}, k=0,1, \ldots, \ell$, depend on $\mu, \nu, m, j, \gamma$ and $\left.\ell\right)$.

Proof. It is clear that in the same way as (2.3) gives (2.4), (2.3) also produces

$$
\lambda_{n+m, \nu}=\sum_{k=-1}^{\ell} \frac{S_{\nu, m, k}}{n^{k}}+O\left(n^{-\ell-1}\right) .
$$

On the other hand, in the analogous way as (2.4) and (2.6) give (2.7), (2.4) and (2.6) also furnish

$$
d_{n+m, \nu}=\sum_{k=0}^{\ell} \frac{Q_{\nu, m, k}}{n^{k}}+O\left(n^{-\ell-1}\right) .
$$


Thus, applying $(1+t)^{\delta}=1+\left(\begin{array}{l}\delta \\ 1\end{array}\right) t+\cdots+\left(\begin{array}{l}\delta \\ \ell\end{array}\right) t^{\ell}+O\left(t^{\ell+1}\right), t \rightarrow 0$, with $\delta=-j$ and $\delta=\gamma$ and combining (2.4), (4.4), (2.7) and (4.5) shows that

$$
d_{n+m, \nu} d_{n, \mu} \lambda_{n+m, \nu}^{-j} \lambda_{n, \mu}^{\gamma}=n^{-j+\gamma} \sum_{k=0}^{\ell} \frac{T_{k}}{n^{k}}+O\left(n^{-\ell-1}\right),
$$

where the coefficients $T_{k}$ as well as the constant involved in the remainder depend on $\mu, \nu, m, j, \gamma$ and $\ell$.

Now, using Taylor's expansion of degree $\ell$ and the fact that by (2.2), (2.4) and (4.4), $c_{n+m, \nu}$ and $c_{n, \mu}$ can be written as $\sum_{k=1}^{\ell} \tilde{S}_{k} n^{-k}$ plus an $O\left(n^{-\ell-1}\right)$ remainder, one can check that (a note that $S_{\mu,-1}=S_{\nu, m,-1}=\pi, S_{\mu, 0}=\pi D_{\mu}$ and $S_{\nu, m, 0}=$ $\pi\left(m+D_{\nu}\right)$ may be helpful $)$

$$
U(n, x) V(n, y)=\sum_{k=0}^{\ell} \frac{B_{k}(x, y)}{n^{k}}+r_{n}(x, y),
$$

where $B_{k}(x, y)$ are polynomials in $x$ and $y$, and $\left|r_{n}(x, y)\right| \leq C n^{-\ell-1}$. Combining (4.6) and (4.7) produces (4.3).

Proposition 4.3. Let $\mu, \nu>-1, m$ be an integer and let $s<\mu+\nu+2$. Then,

$$
\left|L_{s, m}(r, x, y)\right| \leq C x^{s-\mu-3 / 2} y^{\mu+1 / 2}, \quad 0<y<x / 2,
$$

and

$$
\left|L_{s, m}(r, x, y)\right| \leq C y^{s-\nu-3 / 2} x^{\nu+1 / 2}, \quad 2 x<y<1,
$$

with a constant $C$ independent of $0<r<1,0<x<1$ and $0<y<1$.

Proof. The second estimate is the dual form of the first one, hence we concentrate on proving the first estimate for the region $0<y<x / 2$. It is enough to show that given an integer $m$, for $0<r<1,0<x<1,0<y<x / 2$ and $s<\mu+\nu+2$,

$$
\left|\sum_{n=1}^{\infty} r^{n} n^{-s} \psi_{n+m}^{\nu}(x) d_{n, \mu} \lambda_{n, \mu}^{\mu+1 / 2} \cos \left(\lambda_{n, \mu} y\right)\right| \leq C x^{s-\mu-3 / 2}
$$

and the analogous estimate with the exponents $\mu+1 / 2$ and $s-\mu-3 / 2$ replaced by $(\mu+2)+1 / 2$ and $s-(\mu+2)-3 / 2$, respectively. Indeed, using (4.8) and Poisson's integral formula

$$
J_{\mu}(z)=C_{\mu} z^{\mu} \int_{0}^{1}\left(1-t^{2}\right)^{\mu-1 / 2} \cos (z t) d t, \quad \mu>-1 / 2,
$$

gives, for $\mu>-1 / 2$,

$$
\begin{aligned}
& \left|L_{s, m}(r, x, y)\right| \\
& \quad=C_{\mu}\left|\sum_{n=1}^{\infty} r^{n} n^{-s} \psi_{n+m}^{\nu}(x) d_{n, \mu}\left(\lambda_{n, \mu} y\right)^{\mu+1 / 2} \int_{0}^{1}\left(1-t^{2}\right)^{\mu-1 / 2} \cos \left(\lambda_{n, \mu} y t\right) d t\right| \\
& \quad \leq C y^{\mu+1 / 2} \int_{0}^{1}\left(1-t^{2}\right)^{\mu-1 / 2}\left|\sum_{n=1}^{\infty} r^{n} n^{-s} \psi_{n+m}^{\nu}(x) d_{n, \mu} \lambda_{n, \mu}^{\mu+1 / 2} \cos \left(\lambda_{n, \mu} y t\right)\right| d t \\
& \quad \leq C x^{s-\mu-3 / 2} y^{\mu+1 / 2} .
\end{aligned}
$$

In the case $-1<\mu \leq-1 / 2$, applying the identity

$$
J_{\mu}(z)=-J_{\mu+2}(z)+\frac{2(\mu+1)}{z} J_{\mu+1}(z)
$$


gives

$$
\begin{aligned}
L_{s, m}(r, x, y)=-\sum_{n=1}^{\infty} & r^{n} n^{-s} \psi_{n+m}^{\nu}(x) d_{n, \mu}\left(\lambda_{n, \mu} y\right)^{1 / 2} J_{\mu+2}\left(\lambda_{n, \mu} y\right) \\
& +2(\mu+1) \sum_{n=1}^{\infty} r^{n} n^{-s} \psi_{n+m}^{\nu}(x) d_{n, \mu}\left(\lambda_{n, \mu} y\right)^{-1 / 2} J_{\mu+1}\left(\lambda_{n, \mu} y\right) .
\end{aligned}
$$

Now, using Poisson's formula in both summands and applying (4.8) we obtain the result. (In the first sum, after applying Poisson's formula, we need an analogue of (4.8) with the exponent in $\lambda_{n, \mu}$ changed by $\mu+2+1 / 2$. This fact produces the estimate $x^{s-(\mu+2)-3 / 2} y^{\mu+2+1 / 2}$ for the first sum. Finally, the assumption $y \leq x / 2$ gives the required inequality.)

To prove (4.8) (the proof of the estimate similar to (4.8) with the aforementioned replacements follows analogously) we split the sum into two relevant parts. Taking $N=\left[\frac{1}{x}\right]$, let

$$
A=\sum_{n=1}^{N-1} r^{n} n^{-s} \psi_{n+m}^{\nu}(x) d_{n, \mu} \lambda_{n, \mu}^{\mu+1 / 2} \cos \left(\lambda_{n, \mu} y\right)
$$

and

$$
B=\sum_{n=N}^{\infty} r^{n} n^{-s} \psi_{n+m}^{\nu}(x) d_{n, \mu} \lambda_{n, \mu}^{\mu+1 / 2} \cos \left(\lambda_{n, \mu} y\right) .
$$

Using (2.1), (2.2) and (2.5) produces

$$
|A| \leq C x^{1 / 2} \sum_{n=1}^{N-1} n^{-s+\mu+1}\left|J_{\nu}\left(\lambda_{n+m, \nu} x\right)\right| \leq C x^{\nu+1 / 2} \sum_{n=1}^{N-1} n^{-s+\mu+\nu+1} \leq C x^{s-\mu-3 / 2} .
$$

Estimating $B$ we use (2.6), choosing the value of $M$ in a moment. It is then clear that

$$
|B| \leq C \sum_{j=0}^{M} x^{-j}\left(\left|\mathcal{C}_{j}\right|+\left|\mathcal{S}_{j}\right|\right)+G_{M}
$$

where

$$
\left\{\begin{array}{l}
\mathcal{S}_{j} \\
\mathcal{C}_{j}
\end{array}\right\}=\sum_{n=N}^{\infty} r^{n} n^{-s} d_{n+m, \nu} d_{n, \mu} \lambda_{n+m, \nu}^{-j} \lambda_{n, \mu}^{\mu+1 / 2}\left\{\begin{array}{c}
\sin \\
\cos
\end{array}\right\}\left(\lambda_{n+m, \nu} x\right) \cos \left(\lambda_{n, \mu} y\right),
$$

$j=0,1, \ldots, M$, and

$$
G_{M}=\sum_{n=N}^{\infty} n^{-s} d_{n+m, \nu} d_{n, \mu}\left|H_{M}\left(\lambda_{n+m, \nu} x\right)\right| \lambda_{n, \mu}^{\mu+1 / 2} .
$$

If $M$ is such that $M>-s+\mu+1 / 2$, then $G_{M}$ is well controlled. Indeed, using (2.1) and (2.2) we have

$$
\begin{aligned}
G_{M} & \leq C x^{-(M+1)} \sum_{n=N}^{\infty} n^{-(s+M+1 / 2)+\mu} \\
& \leq C x^{-(M+1)} N^{-(s+M+1 / 2)+\mu+1} \\
& \leq C x^{s-\mu-3 / 2} .
\end{aligned}
$$


Taking into account (4.9), to finish the proof of (4.8) it remains to check that both $\left|\mathcal{S}_{j}\right|$ and $\left|\mathcal{C}_{j}\right|$ are bounded by $C x^{s+j-\mu-3 / 2}$. If it happens that $-s+\mu+1 / 2<-1$, then we choose $M=0$, and the required bound for $\left|\mathcal{S}_{j}\right|$ and $\left|\mathcal{C}_{j}\right|$ with $j=0$ immediately follows. Hence, from now on we assume $-s+\mu+1 / 2 \geq-1$ and choose a (uniquely determined) nonnegative integer $M$ such that $M-1 \leq-s+\mu+1 / 2<M$. It follows from Lemmas 4.1 and 4.2 that for given $j=0,1, \ldots, M, \mathcal{S}_{j}$ and $\mathcal{C}_{j}$ are linear combinations, with bounded functions as coefficients, of the (sixteen) series of the form

$$
\sum_{n=N}^{\infty} r^{n} n^{-s-j+\mu+1 / 2} E_{j, \mu+1 / 2, M-j}(n, x, y)\left\{\begin{array}{c}
\sin \\
\cos
\end{array}\right\}(\pi n x)\left\{\begin{array}{c}
\sin \\
\cos
\end{array}\right\}(\pi n y),
$$

where $E_{j, \mu+1 / 2, M-j}(n, x, y)$ is as in Lemma 4.2. It is therefore clear that our task is reduced to estimating the absolute value of each of the series in (4.10) by $C x^{s+j-\mu-3 / 2}$. Given $j=0, \ldots, M$, we use the expression for $E_{j, \mu+1 / 2, M-j}(n, x, y)$ from Lemma 4.2 to show that the absolute value of

$$
R_{j, k}=\sum_{n=N}^{\infty} r^{n} n^{-s-j-k+\mu+1 / 2}\left\{\begin{array}{c}
\sin \\
\cos
\end{array}\right\}(\pi n x)\left\{\begin{array}{l}
\sin \\
\cos
\end{array}\right\}(\pi n y)
$$

is, for $k=0, \ldots, M-j$, bounded by $C x^{s+j-\mu-3 / 2}$ and

$$
\left|\sum_{n=N}^{\infty} r^{n} n^{-s-j+\mu+1 / 2} q_{n}(x, y)\left\{\begin{array}{l}
\sin \\
\cos
\end{array}\right\}(\pi n x)\left\{\begin{array}{l}
\sin \\
\cos
\end{array}\right\}(\pi n y)\right| \leq C x^{s+j-\mu-3 / 2} .
$$

For the term involving $q_{n}(x, y)$, using the fact that $-(s+M+1 / 2)+\mu<-1$ gives

$$
\begin{aligned}
\left|\sum_{n=N}^{\infty} r^{n} n^{-s-j+\mu+1 / 2} q_{n}(x, y)\left\{\begin{array}{c}
\sin \\
\cos
\end{array}\right\}(\pi n x)\left\{\begin{array}{c}
\sin \\
\cos
\end{array}\right\}(\pi n y)\right| & \leq C \sum_{n=N}^{\infty} n^{-(s+M+1 / 2)+\mu} \\
& \leq C x^{s+M-\mu-1 / 2}
\end{aligned}
$$

which is enough for our purpose.

The hypothesis made on $M$ shows that $-s-j-k+\mu+1 / 2>-1$ for $j=0, \ldots, M$ and $k=0, \ldots, M-j$ when $M-1<-s+\mu+1 / 2$, and the same is true for $j=0, \ldots, M$ and $k=0, \ldots, M-j-1$ when $M-1=-s+\mu+1 / 2$. Hence, in these cases,

$$
\begin{aligned}
\left|\sum_{n=1}^{N-1} r^{n} n^{-s-j-k+\mu+1 / 2}\left\{\begin{array}{c}
\sin \\
\cos
\end{array}\right\}(\pi n x)\left\{\begin{array}{l}
\sin \\
\cos
\end{array}\right\}(\pi n y)\right| & \leq C \sum_{n=1}^{N} n^{-s-j-k+\mu+1 / 2} \\
& \leq C x^{s+j+k-\mu-3 / 2} \\
& \leq C x^{s+j-\mu-3 / 2}
\end{aligned}
$$

As a consequence, in (4.11) we can extend the sum to start from $n=1$ and then use Lemma 3.3 to estimate the complete sum. Thus,

$$
\begin{aligned}
\left|\tilde{R}_{j, k}\right| & =\left|\sum_{n=1}^{\infty} r^{n} n^{-s-j-k+\mu+1 / 2}\left\{\begin{array}{l}
\sin \\
\cos
\end{array}\right\}(\pi n x)\left\{\begin{array}{l}
\sin \\
\cos
\end{array}\right\}(\pi n y)\right| \\
& \leq C x^{s+j-\mu-3 / 2} .
\end{aligned}
$$

This completes the estimate of $R_{j, k}, k=0, \ldots, M-j$, except the cases of $R_{j, M-j}$ when $M-1=-s+\mu+1 / 2$ for $j=0, \ldots, M$. In this exceptional case we have 
to show that $\left|R_{j, M-j}\right| \leq C x^{j-M}$; in fact we will prove that $\left|R_{j, M-j}\right| \leq C$. Since, with the assumption $M-1=-s+\mu+1 / 2$,

$$
R_{j, M-j}=\sum_{n=N}^{\infty} \frac{r^{n}}{n}\left\{\begin{array}{l}
\sin \\
\cos
\end{array}\right\}(\pi n x)\left\{\begin{array}{l}
\sin \\
\cos
\end{array}\right\}(\pi n y)
$$

using the fact that

$$
\left\{\begin{array}{l}
\sin \\
\cos
\end{array}\right\}(\pi n x)\left\{\begin{array}{l}
\sin \\
\cos
\end{array}\right\}(\pi n y)
$$

is a linear combination of terms of the form $\sin (\pi n(x \pm y))$ and $\cos (\pi n(x \pm y))$ and that $N=\left[\frac{1}{x}\right] \sim\left[\frac{1}{x+y}\right] \sim\left[\frac{1}{|x-y|}\right]$, it is enough to prove that

$$
\left|\sum_{n=N}^{\infty} \frac{r^{n}}{n}\left\{\begin{array}{l}
\sin \\
\cos
\end{array}\right\}(n t)\right| \leq C
$$

for $0<|t|<3 \pi / 2$, where $N=\left[\frac{1}{|t|}\right]$. The summation by parts formula used with $a_{n}=r^{n} / n$ and

$$
b_{n}=\tilde{D}_{n}(t)=\sum_{k=0}^{n-1}\left\{\begin{array}{l}
\sin \\
\cos
\end{array}\right\}(k t)
$$

(the modified or the conjugate modified Dirichlet kernel) produces

$$
\begin{array}{r}
\left|\sum_{n=N}^{\infty} \frac{r^{n}}{n}\left\{\begin{array}{l}
\sin \\
\cos
\end{array}\right\}(n t)\right| \leq \frac{\left|\tilde{D}_{N}(t)\right|}{N}+(1-r)\left|\sum_{n=N}^{\infty} \frac{r^{n}}{n+1} \tilde{D}_{n+1}(t)\right| \\
+\left|\sum_{n=N}^{\infty} \frac{r^{n}}{n(n+1)} \tilde{D}_{n+1}(t)\right| .
\end{array}
$$

Applying the bound $\left|\tilde{D}_{n}(t)\right| \leq C n$ and summing the geometric series shows that the first and second summands above are $O(1)$. For the third summand we use the estimate $\left|\tilde{D}_{n}(t)\right| \leq C /|t|, 0<|t|<3 \pi / 2$ to majorize this summand by

$$
C|t|^{-1} \sum_{n=N+1}^{\infty} n^{-2} \leq C .
$$

This completes the proof of (4.12) and hence finishes estimating $\mathcal{S}_{M}$ and $\mathcal{C}_{M}$ when $M-1=-s+\mu+1 / 2$. The proof of Proposition 4.3 is completed.

\section{Asymptotics of the KeRnel For COMPARABle VAlues of $x$ AND $y$}

This section contains the proof of an asymptotic estimate of the transplantation kernel for comparable values of $x$ and $y$ along the diagonal $x=y$.

Proposition 5.1. Let $\mu, \nu>-1, m$ be an integer, $0 \leq s<\mu+\nu+2,0<r<1$, $0<x, y<1$ and $x / 2<y<2 x$. If $s=0$, then

$$
\begin{aligned}
\mid L_{0, m}(r, x, y)-u_{m}(x, y) & Q_{r}(\pi(x-y)) \mid \\
\leq C & \left(\frac{1}{x} \log \left(\frac{2 x}{|x-y|}\right)+P_{r}(\pi(x-y))+\frac{1}{2-x-y}\right),
\end{aligned}
$$


where $u_{m}(x, y)$ is a bounded function. If $s>0$, then

$$
\left|L_{s, m}(r, x, y)\right| \leq C \begin{cases}|x-y|^{s-1}, & \text { for } 0<s<1, \\ \log \left(\frac{2 x}{|x-y|}\right), & \text { for } s=1, \\ 1, & \text { for } s>1 .\end{cases}
$$

In both cases $C$ is independent of $r, x$ and $y$.

Proof. We start with considerations that apply to both (5.1) and (5.2). Using (2.6) with $M=1$ to expand $\psi_{n+m}^{\nu}(x)$ and $\psi_{n}^{\mu}(y)$, and taking $N=\left[\frac{1}{x}\right] \sim\left[\frac{1}{y}\right]$, we write

$$
L_{s, m}(r, x, y)=F(x, y)+\sum_{j, l=0}^{1} x^{-j} y^{-l} O_{j, l}(x, y)+J_{1}(x, y)+J_{2}(x, y)+G(x, y) \text {, }
$$

where

$$
F(x, y)=\sum_{n=1}^{N-1} r^{n} n^{-s} \psi_{n+m}^{\nu}(x) \psi_{n}^{\mu}(y) .
$$

Also, for the remainder sum that starts from $n=N$, the $O_{j, l}$ terms capture the part that comes from the main parts of the aforementioned expansions and are the sums of four terms of the form

$$
D_{j, l} \sum_{n=N}^{\infty} r^{n} n^{-s} d_{n+m, \nu} d_{n, \mu} \lambda_{n+m, \nu}^{-j} \lambda_{n, \mu}^{-l}\left\{\begin{array}{c}
\sin \\
\cos
\end{array}\right\}\left(\lambda_{n+m, \nu} x\right)\left\{\begin{array}{l}
\sin \\
\cos
\end{array}\right\}\left(\lambda_{n, \mu} y\right)
$$

( $D_{j, l}$ is a product of $A_{\nu, j}$ or $B_{\nu, j}$ and $A_{\mu, l}$ or $B_{\mu, l}$ depending on the choice of the sine or cosine), $J_{1}$ gathers the part that comes from the main parts of the first expansion and the remainder of the second one. Hence its absolute value is bounded by

$$
\begin{aligned}
\left|J_{1}(x, y)\right| \leq & C\left|\sum_{1}^{2} \sum_{n=N}^{\infty} r^{n} n^{-s} d_{n+m, \nu} d_{n, \mu} H_{1}\left(\lambda_{n+m, \nu} x\right)\left\{\begin{array}{l}
\sin \\
\cos
\end{array}\right\}\left(\lambda_{n, \mu} y\right)\right| \\
& +C y^{-1}\left|\sum_{1}^{2} \sum_{n=N}^{\infty} r^{n} n^{-s} \lambda_{n, \mu}^{-1} d_{n+m, \nu} d_{n, \mu} H_{1}\left(\lambda_{n+m, \nu} x\right)\left\{\begin{array}{l}
\sin \\
\cos
\end{array}\right\}\left(\lambda_{n, \mu} y\right)\right|
\end{aligned}
$$

(the sign $\sum_{1}^{2}$ indicates that we add two series, one for the choice of the sine another for the cosine), $J_{2}$ acts as $J_{1}$ but with the position of the both expansions switched, its absolute value is controlled by

$$
\begin{aligned}
&\left|J_{2}(x, y)\right| \leq C\left|\sum_{1}^{2} \sum_{n=N}^{\infty} r^{n} n^{-s} d_{n+m, \nu} d_{n, \mu}\left\{\begin{array}{l}
\sin \\
\cos
\end{array}\right\}\left(\lambda_{n+m, \nu} x\right) H_{1}\left(\lambda_{n, \mu} y\right)\right| \\
&+C x^{-1}\left|\sum_{1}^{2} \sum_{n=N}^{\infty} r^{n} n^{-s} \lambda_{n+m, \nu}^{-1} d_{n+m, \nu} d_{n, \mu}\left\{\begin{array}{c}
\sin \\
\cos
\end{array}\right\}\left(\lambda_{n+m, \nu} x\right) H_{1}\left(\lambda_{n, \mu} y\right)\right|
\end{aligned}
$$

and, eventually, $G$ captures the part that comes from the remainders,

$$
G(x, y)=\sum_{n=N}^{\infty} r^{n} n^{-s} d_{n+m, \nu} d_{n, \mu} H_{1}\left(\lambda_{n+m, \nu} x\right) H_{1}\left(\lambda_{n, \mu} y\right) .
$$

We will now separately analyze each of the eight summands in the above decomposition of $L_{s, m}$ and bound them, with the exception of the $O_{0,0}$ in the $s=0$ case, by one of the terms on the right of (5.1) or (5.2). In the exceptional case, using 
Lemma 4.1 and Lemma 4.2, we will write each of the terms included in $O_{0,0}$ in a more regular form and then majorize them, whenever possible, by the first or third term on the right of (5.1). Such a reduction will finally lead, after extending the summation in the appropriate sums to the set of all positive integers, to the sum of the form

$$
u_{m}(x, y) Q_{r}(\pi(x-y))+v_{m}(x, y) P_{r}(\pi(x-y)),
$$

with bounded functions $u_{m}$ and $v_{m}$.

For $F(x, y)$, using (2.1), (2.2) and (2.5) and taking into account that $-s+\nu+$ $\mu+1>-1$, we have

$$
|F(x, y)| \leq C x^{\nu+1 / 2} y^{\mu+1 / 2} \sum_{n=1}^{N-1} n^{-s+\nu+\mu+1} \leq C x^{\nu+\mu+1} N^{-s+\nu+\mu+2} \leq C x^{s-1},
$$

and this is always controlled by the right sides of (5.1) or (5.2).

For $J_{1}(x, y)$ (the same reasoning works for $J_{2}(x, y)$ ), using $H_{1}(x)=O\left(x^{-2}\right)$, $x \geq 1$, and again (2.1) and (2.2), shows that

$$
\begin{aligned}
\left|J_{1}(x, y)\right| & \leq C x^{-2}\left(\sum_{n=N}^{\infty} n^{-(s+2)}+y^{-1} \sum_{n=N}^{\infty} n^{-(s+3)}\right) \\
& \leq C x^{-2}\left(N^{-(s+1)}+y^{-1} N^{-(s+2)}\right) \leq C x^{s-1},
\end{aligned}
$$

which is good for our bounds. In a similar way we show that

$$
|G(x, y)| \leq C(x y)^{-2} \sum_{n=N}^{\infty} n^{-(s+4)} \leq C(x y)^{-2} N^{-(s+3)} \leq C x^{s-1} .
$$

The remainder of the proof is concerned with a more delicate analysis of the $O_{j, l}$ terms. We start with the $O_{1,1}$ term. It is clear that, for $s \geq 0$,

$$
\left|x^{-1} y^{-1} O_{1,1}(x, y)\right| \leq C x^{-2} \sum_{n=N}^{\infty} n^{-(s+2)} \leq C x^{-2} N^{-(s+1)} \leq C x^{s-1} .
$$

Exactly the same bound is obtained for $\left|x^{-1} O_{1,0}(x, y)\right|$ and $\left|y^{-1} O_{0,1}(x, y)\right|$ if $s>0$, which is appropriate for (5.2). In the case $s=0$, using Lemma 4.1 and Lemma 4.2 with $j=1$ and $\gamma=\ell=0$, the estimate for $x^{-1} O_{1,0}(x, y)$ follows once we show that

$$
\left|\sum_{n=N}^{\infty} \frac{r^{n}}{n} E_{1,0,0}(n, x, y)\left\{\begin{array}{l}
\sin \\
\cos
\end{array}\right\}(\pi n x)\left\{\begin{array}{l}
\sin \\
\cos
\end{array}\right\}(\pi n y)\right| \leq C \log \left(\frac{2 x}{|x-y|}\right),
$$

where $E_{1,0,0}(n, x, y)$ is as in (4.3). The form of $E_{1,0,0}$ reduces this task to showing the estimates

$$
\left|\sum_{n=N}^{\infty} \frac{r^{n}}{n}\left\{\begin{array}{l}
\sin \\
\cos
\end{array}\right\}(\pi n x)\left\{\begin{array}{l}
\sin \\
\cos
\end{array}\right\}(\pi n y)\right| \leq C \log \left(\frac{2 x}{|x-y|}\right)
$$

and

$$
\left|\sum_{n=N}^{\infty} \frac{r^{n}}{n} q_{n}(x, y)\left\{\begin{array}{c}
\sin \\
\cos
\end{array}\right\}(\pi n x)\left\{\begin{array}{c}
\sin \\
\cos
\end{array}\right\}(\pi n y)\right| \leq C,
$$

where $\left|q_{n}(x, y)\right| \leq C n^{-1}$. The last series is absolutely convergent, and the bound follows. To show (15.3) , we start by denoting $N_{1}=\left[\frac{1}{x+y}\right]$ and $N_{2}=\left[\frac{1}{|x-y|}\right]$. Then 
the left side of (5.3) is bounded by $C\left(J_{0}+\left|J_{1}\right|+\left|J_{2}\right|\right)$, where $J_{0}=\sum_{N_{1}}^{N_{2}} \frac{1}{n}$ and $J_{1}$ and $J_{2}$ are of the form

$$
J_{1}=\sum_{n=N_{1}}^{\infty} \frac{r^{n}}{n}\left\{\begin{array}{l}
\sin \\
\cos
\end{array}\right\}(\pi n(x+y)), \quad J_{2}=\sum_{n=N_{2}}^{\infty} \frac{r^{n}}{n}\left\{\begin{array}{l}
\sin \\
\cos
\end{array}\right\}(\pi n(x-y)) .
$$

It is easy to check that

$$
\sum_{n=N_{1}}^{N_{2}} \frac{1}{n} \leq C \log \left(\frac{x+y}{|x-y|}\right) \leq C \log \left(\frac{2 x}{|x-y|}\right) .
$$

Using (4.12) (recall that $|x-y| \leq 1$ ) we get $\left|J_{2}\right| \leq C$. To estimate $\left|J_{1}\right|$ note that if $0<x, y<1$ and $x / 2<y<2 x$, then $0<x, y<3 / 4$ or $3 / 8<x, y<1$. In the first case $x+y<3 / 2$ and we can use (4.12) to get $\left|J_{1}\right| \leq C$. In the second case we apply the change of variables $x=1-u$ and $y=1-v$. Then, it is clear that $0<u, v<5 / 8$ and $u+v \leq 3 / 2$, and we can apply (4.12) to obtain

$$
\left|\sum_{n=N_{1}}^{\infty} \frac{r^{n}}{n}\left\{\begin{array}{c}
\sin \\
\cos
\end{array}\right\}(\pi n(x+y))\right|=\left|\sum_{n=N_{1}}^{\infty} \frac{r^{n}}{n}\left\{\begin{array}{l}
\sin \\
\cos
\end{array}\right\}(\pi n(u+v))\right| \leq C .
$$

For $\left|y^{-1} O_{0,1}(x, y)\right|$ we argue in the same way. Moreover, taking into account that $x \sim y$, we end up with the same estimate for this term. Summarizing, for $s=0$,

$$
\left|x^{-1} O_{1,0}(x, y)\right|+\left|y^{-1} O_{0,1}(x, y)\right| \leq \frac{C}{x} \log \left(\frac{2 x}{|x-y|}\right),
$$

which is appropriate for (5.1).

It remains to analyze the $O_{0,0}(x, y)$ term. If $s>1$ there is no problem because the four series included in $O_{0,0}(x, y)$ are absolutely convergent, hence $\left|O_{0,0}(x, y)\right| \leq C$ follows which is the correct bound for (5.2) in this case. If $s=1$, following the steps given for $O_{1,0}(x, y)$ with $s=0$ (using $E_{0,0,0}(n, x, y)$ instead of $E_{1,0,0}(n, x, y)$ ), we obtain $\left|O_{0,0}(x, y)\right| \leq C \log \left(\frac{2 x}{|x-y|}\right)$ which is the bound for $L_{1, m}(r, x, y)$. Consider the case $0 \leq s<1$. Again using Lemma 4.1 and Lemma 4.2 with $j=\gamma=0$ and $\ell=1$ shows that $O_{0,0}(x, y)$ is the sum of four terms of the form

$$
D_{0,0} u(x) v(y) \sum_{n=N}^{\infty} r^{n} n^{-s} E_{0,0,1}(n, x, y)\left\{\begin{array}{c}
\sin \\
\cos
\end{array}\right\}(\pi n x)\left\{\begin{array}{c}
\sin \\
\cos
\end{array}\right\}(\pi n y),
$$

or, after applying trigonometric identities and expanding $E_{0,0,1}(n, x, y)$, the sum of four terms of the form

$$
\tilde{u}(x) \tilde{v}(y) \sum_{n=N}^{\infty} r^{n} n^{-s}\left(A_{0}+\frac{A_{1}(x, y)}{n}+q_{n}(x, y)\right)\left\{\begin{array}{l}
\sin \\
\cos
\end{array}\right\}(\pi n(x \pm y)) .
$$

Here $A_{0}$ is a constant, $A_{1}(x, y)$ is a polynomial in $x$ and $y$ of degree 1 , and $\left|q_{n}(x, y)\right| \leq C n^{-2}$ for $0<x, y<1$. We will now analyze each of the three expressions resulting from (5.4). The estimate for the remainder term is immediate since, for $0 \leq s<1$, using $\left|q_{n}(x, y)\right| \leq C n^{-2}$ gives

$$
\left|\sum_{n=N}^{\infty} r^{n} n^{-s} q_{n}(x, y)\left\{\begin{array}{l}
\sin \\
\cos
\end{array}\right\}(\pi n(x \pm y))\right| \leq C \sum_{n=1}^{\infty} n^{-s-2} \leq C,
$$


which is enough for our purpose. The same bound occurs for the term resulting from considering $A_{1}(x, y) n^{-1}$ when $0<s<1$. Keeping for a moment the assumption $0<s<1$ note that since

$$
\left|\sum_{n=1}^{N-1} r^{n} n^{-s} A_{0}\left\{\begin{array}{c}
\sin \\
\cos
\end{array}\right\}(\pi n(x \pm y))\right| \leq C \sum_{n=1}^{N-1} n^{-s} \leq C x^{s-1},
$$

in the term involving $A_{0}$ we can extend the summation to $n=1$. Therefore, the conclusion of the estimate for $O_{0,0}(x, y)$ (with $0<s<1$ ) reduces to checking that

$$
\left|\sum_{n=1}^{\infty} r^{n} n^{-s}\left\{\begin{array}{c}
\sin \\
\cos
\end{array}\right\}(\pi n(x \pm y))\right| \leq C|x-y|^{s-1} .
$$

Since $|x-y| \leq 1$, the minus sign case follows from Lemma 3.3 For the sum with the plus sign consider the cases $0<x, y<3 / 4$ and $3 / 8<x, y<1$. In the first case we use Lemma 3.3 and the obvious inequality $|x-y| \leq|x+y|$. The second case is again treated by using the change of variables $x=1-u$ and $y=1-v$. Then Lemma 3.3 gives

$$
\begin{aligned}
\left|\sum_{n=1}^{\infty} r^{n} n^{-s}\left\{\begin{array}{c}
\sin \\
\cos
\end{array}\right\}(\pi n(x+y))\right| & =\left|\sum_{n=1}^{\infty} r^{n} n^{-s}\left\{\begin{array}{c}
\sin \\
\cos
\end{array}\right\}(\pi n(u+v))\right| \\
& \leq C|u+v|^{s-1} \leq C|u-v|^{s-1}=C|x-y|^{s-1} .
\end{aligned}
$$

This concludes the proof of the estimate $\left|O_{0,0}(x, y)\right| \leq C|x-y|^{s-1}$ in the case $0<s<1$, hence the proof of (5.2).

We are therefore left with the case $s=0$ and the terms resulting from the main terms in (5.4). For the term involving $A_{1}(x, y) n^{-1}$ note that $\tilde{u}(x) \tilde{v}(y) A_{1}(x, y)$ is a bounded function on $0<x, y<1$, hence our task reduces to already proved estimate (5.3). Finally, consider the term involving $A_{0}$. It is possible to extend the summation of the series involving $A_{0}$ in (5.4) from $n=1$ since

$$
\left|\sum_{n=1}^{N-1} r^{n}\left\{\begin{array}{l}
\sin \\
\cos
\end{array}\right\}(\pi n(x \pm y))\right| \leq C \sum_{n=1}^{N-1} 1 \leq C x^{-1}
$$

Now, the series with the plus sign and summation starting from $n=1$ are easily treated. Indeed, assuming $0<x, y<3 / 4$, we apply Lemma 3.3 to obtain

$$
\left|\sum_{n=1}^{\infty} r^{n}\left\{\begin{array}{l}
\sin \\
\cos
\end{array}\right\}(\pi n(x+y))\right| \leq C(x+y)^{-1} \leq C x^{-1} .
$$

Assuming $3 / 8<x, y<1$, write $x=1-u, y=1-v$. Then $0<u+v<3 / 2$, hence

$$
\begin{aligned}
\left|\sum_{n=1}^{\infty} r^{n}\left\{\begin{array}{l}
\sin \\
\cos
\end{array}\right\}(\pi n(x+y))\right| & =\left|\sum_{n=1}^{\infty} r^{n}\left\{\begin{array}{l}
\sin \\
\cos
\end{array}\right\}(\pi n(u+v))\right| \\
& \leq C(u+v)^{-1}=C(2-x-y)^{-1} .
\end{aligned}
$$

We now conclude by stating that what is left from $L_{0, m}(r, x, y)$ (and has not already been estimated by one of the terms on the right of (5.1)) is the expression

$$
A_{0,1} \tilde{u}_{1}(x) \tilde{v}_{1}(y) \sum_{n=1}^{\infty} r^{n} \sin (\pi n(x-y))+A_{0,2} \tilde{u}_{2}(x) \tilde{v}_{2}(y) \sum_{n=1}^{\infty} r^{n} \cos (\pi n(x-y)) .
$$


Estimating the absolute value of the second term results in bringing $C P_{r}(\pi(x-y))$ on the right of (5.1). Consideration of the first term results in choosing $u_{m}(x, y)=$ $A_{0,1} \tilde{u}_{1}(x) \tilde{v}_{1}(y)$. This finishes the analysis of the $O_{0,0}$ term in the case $s=0$, hence concludes the proof of Proposition 5.1.

\section{Proof of Theorem 1.1}

We start by noting that $T_{r} f(x)$ is well defined for every $x \in(0,1)$ : this is a simple consequence of (2.8) and (2.9).

The dominated convergence theorem together with (2.9) and (2.8) show that for given $x$ and $r$

$$
T_{r} f(x)=\int_{0}^{1} L_{0, m}(r, x, y) f(y) d y .
$$

Take $f \in L^{p, a}$. For the proof of (1.2) it is sufficient to verify that the quantity

$$
\int_{0}^{1}\left|\int_{0}^{1} L_{0, m}(r, x, y) f(y) d y\right|^{p} x^{a} d x
$$

is bounded by $C\|f\|_{p, a}^{p}$. To check this, split the inner integration onto the intervals $(0, x / 2),(x / 2, \min \{3 x / 2,1\})$ and $(\min \{3 x / 2,1\}, 1)$, and consider each of the resulting integrals separately. For the first and third integrals the result immediately follows by using the estimates of Proposition 4.3 with $s=0$, Hardy's inequalities (2.12) and (2.13), and the assumption (1.1). For the second integral we apply the estimate of Proposition 5.1 and then an argument similar to that of [5], pp. $38-$ 39. Thus we need to bound the quantities

$$
\begin{gathered}
\int_{0}^{1}\left(\int_{x / 2}^{\min \{3 x / 2,1\}} \frac{|f(y)|}{x} \log \left(\frac{2 x}{|x-y|}\right) d y\right)^{p} x^{a} d x \\
\int_{0}^{1}\left(\int_{x / 2}^{\min \{3 x / 2,1\}} P_{r}(\pi(x-y))|f(y)| d y\right)^{p} x^{a} d x \\
\int_{0}^{1}\left(\int_{x / 2}^{\min \{3 x / 2,1\}} \frac{|f(y)|}{2-x-y} d y\right)^{p} x^{a} d x,
\end{gathered}
$$

and

$$
\int_{0}^{1}\left|\int_{x / 2}^{\min \{3 x / 2,1\}} u_{m}(x, y) Q_{r}(\pi(x-y)) f(y) d y\right|^{p} x^{a} d x,
$$

by $\mathrm{C}\|f\|_{p, a}^{p}$ with $C$ independent of $0<r<1$ and $f$. For (6.2), (6.3) and (6.5) we copy the argument of [5, p. 39]. The checking the required bound for (6.4) reduces to showing the inequality

$$
\int_{0}^{1}\left(\int_{x / 2}^{\min \{3 x / 2,1\}} \frac{h(y)}{2-x-y} d y\right)^{p} d x \leq C \int_{0}^{1} h(y)^{p} d y,
$$

where $h$ is any nonnegative function on $(0,1)$. This in order, by enlarging the region of the inner integration to $(0,1)$ and changing the variables, $u=1-x, v=1-y$, reduces to verifying that

$$
\int_{0}^{1}\left(\int_{0}^{1} \frac{g(v)}{u+v} d v\right)^{p} d u \leq C \int_{0}^{1} g(v)^{p} d v
$$


where $g \geq 0$. We have

$$
\begin{aligned}
& \left(\int_{0}^{1}\left(\int_{0}^{1} \frac{g(v)}{u+v} d v\right)^{p} d u\right)^{1 / p} \\
\leq & \left(\int_{0}^{1}\left(\int_{0}^{u} \frac{g(v)}{u+v} d v\right)^{p} d u\right)^{1 / p}+\left(\int_{0}^{1}\left(\int_{u}^{1} \frac{g(v)}{u+v} d v\right)^{p} d u\right)^{1 / p} \\
\leq & \left(\int_{0}^{1}\left(\frac{1}{u} \int_{0}^{u} g(v) d v\right)^{p} d u\right)^{1 / p}+\left(\int_{0}^{1}\left(\int_{u}^{1} \frac{g(v)}{v} d v\right)^{p} d u\right)^{1 / p} \\
\leq & C\left(\int_{0}^{1} g(v)^{p} d v\right)^{1 / p} .
\end{aligned}
$$

For the last inequality, Hardy's inequalities (2.12) and (2.13) were used (with $a=$ $-p$ and $a=0$ correspondingly). This finishes the proof of estimating (6.1) by $C\|f\|_{p, a}^{p}$ and thus (1.2).

To prove the existence of a function $T f \in L^{p, a}$ such that (1.3) is satisfied, it is sufficient to show that for $\varepsilon>0$ there is an $0<r_{o}<1$ such that for $r_{o}<$ $r<t<1,\left\|T_{r} f-T_{t} f\right\|_{p, a} \leq C \varepsilon$ with $C$ independent of $\varepsilon$. Given $\varepsilon>0$ and a function $f \in L^{p, a}$, take $h \in C_{c}^{\infty}(0,1)$ such that $\|f-h\|_{p, a}<\varepsilon$. Then, by using (1.2), $\left\|T_{r} f-T_{r} h\right\|_{p, a} \leq C \varepsilon$. Using (2.10), (2.11) with $k$ sufficiently large and Minkowski's inequality shows that there exists an $r_{o}$ such that $\left\|T_{r} h-T_{t} h\right\|_{p, a} \leq \varepsilon$ for $r_{o}<r, t<1$. This, together with the triangle inequality, completes the proof of the estimate $\left\|T_{r} f-T_{t} f\right\|_{p, a} \leq C \varepsilon$ and thus the existence of $T f$ satisfying (1.3). Clearly enough, the bound $\|T f\|_{p, a} \leq C\|f\|_{p, a}$ is a consequence of (1.2) and (1.3).

Finally we prove (1.5). It follows by (1.4), (2.10) and Hölder's inequality that for any fixed $n, n=1,2, \ldots$, the mapping $g \rightarrow c_{n}^{\nu}(g)$ is a bounded functional on $L^{p, a}$. Therefore, by using (1.3),

$$
c_{n}^{\nu}(T f)=\lim _{r \rightarrow 1^{-}} c_{n}^{\nu}\left(T_{r} f\right),
$$

which gives (1.5) once we note that $c_{n}^{\nu}\left(T_{r} f\right)$ equals 0 for $1 \leq n \leq m$ and $r^{n} c_{n-m}^{\mu}(f)$ for $\max \{1, m+1\} \leq n<\infty$. The last statement is a consequence of the fact that

$$
\int_{0}^{1}\left(\sum_{j=1}^{\infty} r^{j}\left|c_{j}^{\mu}(f)\right|\left|\psi_{j+m}^{\nu}(x)\right|\right)\left|\psi_{n}^{\nu}(x)\right| d x<\infty,
$$

which follows by (2.8) and (2.9), and then the dominated convergence theorem shows that

$$
\int_{0}^{1} \psi_{n}^{\nu}(x) T_{r} f(x) d x=r^{n} \begin{cases}0, & 1 \leq n \leq m, \\ c_{n-m}^{\mu}(f), & \max \{1, m+1\} \leq n<\infty .\end{cases}
$$

This finishes the proof of (1.5) hence Theorem 1.1

\section{Proof of Theorem 1.3}

The fact that the series defining $T_{r} f(x)$ converges is again a consequence of (2.8) and (2.9). 
The checking of (1.7) is accomplished once we show that each of the quantities

$$
\begin{gathered}
\left(\int_{0}^{1}\left(\int_{0}^{x / 2}\left|f(y) L_{s, m}(r, x, y)\right| d y\right)^{q} x^{a q} d x\right)^{1 / q}, \\
\left(\int_{0}^{1}\left(\int_{x / 2}^{\min \{3 x / 2,1\}}\left|f(y) L_{s, m}(r, x, y)\right| d y\right)^{q} x^{a q} d x\right)^{1 / q}, \\
\left(\int_{0}^{1}\left(\int_{\min \{3 x / 2,1\}}^{1}\left|f(y) L_{s, m}(r, x, y)\right| d y\right)^{q} x^{a q} d x\right)^{1 / q},
\end{gathered}
$$

is bounded by

$$
C\left(\int_{0}^{1}|f(x)|^{p} x^{a p} d x\right)^{1 / p}
$$

Considering (7.1) we use the estimate of $L_{s, m}$ from Proposition 4.3 to conclude that (7.1) is bounded by

$$
\left(\int_{0}^{1}\left(\int_{0}^{x / 2}|f(y)| y^{\mu+1 / 2} d y\right)^{q} x^{(a+s-\mu-3 / 2) q} d x\right)^{1 / q} .
$$

Thus our task reduces to showing the inequality

$$
\left(\int_{0}^{1}\left(\int_{0}^{x / 2}|g(y)| d y U(x)\right)^{q} d x\right)^{1 / q} \leq C\left(\int_{0}^{1}|g(x) V(x)|^{p} d x\right)^{1 / p},
$$

with $U(x)=x^{a+s-\mu-3 / 2}$ and $V(x)=x^{a-\mu-1 / 2}$. We apply a general form of Hardy's inequality; cf. for instance [5, Theorem (9.17)]. The condition to be verified there is

$$
\left(\int_{t}^{1} U(x)^{q} d x\right)^{1 / q}\left(\int_{0}^{t} V(x)^{-p^{\prime}} d x\right)^{1 / p^{\prime}} \leq A
$$

with a constant $A$ independent of $0<t<1$. This is achieved by considering three cases depending on the sign of $(a+s-\mu-3 / 2) q+1$, writing down the values of involved integrals, and observing that the resulting function of $t$ is bounded on $0<t<1$. Clearly, to assure the convergence of the second integral in (17.4) the condition $-(a-\mu-1 / 2) p^{\prime}>-1$ is necessary, but this is just the right side of our assumption (1.6).

For (7.2) we first apply Proposition 4.3 to bound (7.2) by

$$
\left(\int_{0}^{1}\left(\int_{x / 2}^{\min \{3 x / 2,1\}}|f(y)||x-y|^{s-1} d y x^{a}\right)^{q} d x\right)^{1 / q}
$$

and then use [5, Lemma (9.6)] with the weight $w(x)=x^{a}$. The assumption (9.7) of that lemma is satisfied by any power weight, and the conclusion says that (7.5) is bounded by $C\left(\int_{0}^{1}|f(x)|^{p} x^{a p} d x\right)^{1 / p}$.

Considering (7.3) we again use the estimate of $L_{s, m}$ from Proposition 4.3, concluding this time that (7.3) is bounded by

$$
\left(\int_{0}^{1}\left(\int_{\min \{3 x / 2,1\}}^{1}|f(y)| y^{s-\nu-3 / 2} d y\right)^{q} x^{(a+\nu+1 / 2) q} d x\right)^{1 / q}
$$

and then reducing the problem to showing the inequality

$$
\left(\int_{0}^{1}\left(\int_{\min \{3 x / 2,1\}}^{1}|g(y)| d y U(x)\right)^{q} d x\right)^{1 / q} \leq C\left(\int_{0}^{1}|g(x) V(x)|^{p} d x\right)^{1 / p},
$$


with $U(x)=x^{a+\nu+1 / 2}$ and $V(x)=x^{-s+\nu+3 / 2+a}$. We now apply [5, Theorem (9.18)], where the condition to be verified is

$$
\left(\int_{0}^{t} U(x)^{q} d x\right)^{1 / q}\left(\int_{t}^{1} V(x)^{-p^{\prime}} d x\right)^{1 / p^{\prime}} \leq A, \quad 0<t<1 .
$$

Again we consider three cases that depend on the sign of $-(-s+\nu+3 / 2+a) p^{\prime}+1$, we write down the values of involved integrals, and we observe that the resulting function of $t$ is bounded on $0<t<1$. To assure the convergence of the first integral in (7.6) the condition $(a+\nu+1 / 2) q>-1$ is necessary, and this is the left side of (1.6). This finishes estimating (7.3), hence the proof of (1.7).

The argument for existence of an operator $T$ satisfying (1.8) is completely similar to that of the proof of the analogous statement in Theorem 1.1. cf. Section 6 . Hence we will not repeat it.

For the proof of (1.10) note that for any fixed $n, n=1,2, \ldots$, by (1.9), (2.10) and Hölder's inequality it follows that the mapping $g \rightarrow c_{n}^{\nu}(g)$ is a bounded functional on $L^{q, a q}$. Therefore, by using (1.8),

$$
c_{n}^{\nu}(T f)=\lim _{r \rightarrow 1^{-}} c_{n}^{\nu}\left(T_{r} f\right) .
$$

It is now sufficient to observe that $c_{n}^{\nu}\left(T_{r} f\right)$ is equal to 0 for $1 \leq n \leq m$ and $r^{n}(n-m)^{-s} c_{n-m}^{\mu}(f)$ for $\max \{1, m+1\} \leq n<\infty$. The last statement is justified by the fact that

$$
\int_{0}^{1}\left(\sum_{j=1}^{\infty} r^{j} j^{-s}\left|c_{j}^{\mu}(f)\right|\left|\psi_{j+m}^{\nu}(x)\right|\right)\left|\psi_{n}^{\nu}(x)\right| d x<\infty,
$$

which follows by (2.8) and (2.9), and then the dominated convergence theorem shows that

$$
\int_{0}^{1} \psi_{n}^{\nu}(x) T_{r} f(x) d x=r^{n} \begin{cases}0, & 1 \leq n \leq m, \\ (n-m)^{-s} c_{n-m}^{\mu}(f), & \max \{1, m+1\} \leq n<\infty .\end{cases}
$$

This finishes the proof of (1.10), hence Theorem 1.3

\section{Proof of Theorem 1.5}

We will use the following multiplier theorem for the sine expansions, which is a consequence of [6, Theorem (9.17)] (cf. also [5, Theorem (13.1)]).

Theorem 8.1. Let $1<p<\infty, 1 \leq t \leq \infty, \lambda>\max \{1 / t,|1 / p-1 / 2|\}$ or $\lambda=t=1$, $m \in M(t, \lambda),|a|<p \lambda,(a+1) / p$ not an integer and

$$
\max \{-1,-1+p(-\lambda+1 / 2)\}<a<\min \{-1+p(\lambda+1 / 2),-1+p(\lambda+1-1 / t)\} .
$$

Then, for $f(x)=\sum_{j=1}^{\infty} d_{j} \sin (\pi j x)$, where $d_{j}=0$ for all but a finite number of $j$ 's and $d_{j}=0$ for $j=1,2, \ldots[(a+1) / p]-1$,

$$
\left\|\sum_{n=1}^{\infty} m_{n} d_{n} \sin (\pi n x)\right\|_{p, a} \leq C B(m, t, \lambda)\left\|\sum_{n=1}^{\infty} d_{n} \sin (\pi n x)\right\|_{p, a},
$$

with $C$ independent of $m, t, \lambda$ and $f$. 
This theorem is obtained from Theorem (9.17) of [6] by taking $a_{1}=a, a_{2}=0$, extending the sequence $\left\{m_{j}\right\}_{j=1}^{\infty}$ to the sequence $\left\{m_{j}^{*}\right\}_{j=-\infty}^{\infty}$ by setting $m_{-j}^{*}=m_{j}$ for $j<0$ and $m_{0}^{*}=0$ (so that $m^{*} \in M^{*}(t, \lambda)$ in the notation of [6]), restricting the class $S_{l_{1}+l_{2}-1}$ that appears in [6. Theorem (9.17)] to the class of odd polynomials from $S_{l_{1}+l_{2}-1}, f(x)=\sum_{|j| \leq N} d_{j} e^{i j x}, d_{-j}=d_{j}$, changing the variable, $(-\pi, \pi) \in$ $x \rightarrow x / \pi$, and, finally, replacing the weight $W(x)=\left|1-e^{-i x}\right|^{a_{1}}$ by $x^{a}$ (which is comparable with $W(x / \pi)$ on $(0,1))$.

Proving Theorem 1.5 we first note that the assumption $-1<a<2 p-1$, a part of (1.11), implies $0<(a+1) / p<2$. Hence, in what follows we use Theorem 8.1 for sequences $d_{j}$ without the restriction on vanishing of the first $[(a+1) / p]-1$ terms. To prove (1.12) we apply Theorem 1.1 with $1 / 2$ and $\nu$ as $\mu$ and $\nu$ to obtain

$$
\left\|\sum_{j=1}^{\infty} m_{j} d_{j} \psi_{j}^{\nu}\right\|_{p, a} \leq C\left\|\sum_{j=1}^{\infty} m_{j} d_{j} \psi_{j}^{1 / 2}\right\|_{p, a}
$$

as a limit case of (1.2) since we have only a finite number of summands. The required assumption (1.1) is included in (1.11). In the same way we apply Theorem 1.1 with $\nu$ and $1 / 2$ as $\mu$ and $\nu$ to obtain

$$
\left\|\sum_{j=1}^{\infty} d_{j} \psi_{j}^{1 / 2}\right\|_{p, a} \leq C\left\|\sum_{j=1}^{\infty} d_{j} \psi_{j}^{\nu}\right\|_{p, a}
$$

as a limit case of (1.2); in this case the required assumption (1.1) is also included in (1.11). Finally, the concluding inequality

$$
\left\|\sum_{j=1}^{\infty} m_{j} d_{j} \sin (\pi j x)\right\|_{p, a} \leq C B(m, t, \lambda)\left\|\sum_{j=1}^{\infty} d_{j} \sin (\pi j x)\right\|_{p, a}
$$

follows from Theorem 8.1 since the conditions imposed on $a$ in this theorem are weaker than conditions on $a$ assumed in Theorem 1.5. This concludes the proof of Theorem 1.5.

\section{Proof of Corollaries 1.2 and 1.4}

Since the proofs of both corollaries are completely analogous, we furnish the proof of Corollary 1.2 only.

Let $f \in C_{c}^{\infty}(0,1)$. The pointwise convergence of the series $\sum_{n=1}^{\infty} c_{n}^{\mu}(f) \psi_{n+m}^{\nu}(x)$ follows from (2.8) and (2.11) taken with $k$ sufficiently large. This shows that we deal with a well-defined operator. Further, since $T_{r} f \rightarrow T f$ in $L^{p, a}$, we can choose a sequence $0<r_{1}<r_{2}<\ldots, r_{j} \rightarrow 1^{-}$, such that $\lim _{j \rightarrow \infty} T_{r_{j}} f(x)=T f(x)$ for almost every $x \in(0,1)$. On the other hand, for every $x \in(0,1)$,

$$
\begin{aligned}
\lim _{r \rightarrow 1^{-}} T_{r} f(x) & =\lim _{r \rightarrow 1^{-}} \sum_{n=1}^{\infty} r^{n} c_{n}^{\mu}(f) \psi_{n+m}^{\nu}(x) \\
& =\sum_{n=1}^{\infty} c_{n}^{\mu}(f) \psi_{n+m}^{\nu}(x) .
\end{aligned}
$$

The last equation is justified by the fact that the last series is absolutely convergent, which is implied by (2.11) and (2.8). Thus we checked that for $f \in C_{c}^{\infty}(0,1)$,

$$
T f(x)=\sum_{n=1}^{\infty} c_{n}^{\mu}(f) \psi_{n+m}^{\nu}(x), \quad x \text {-a.e. }
$$


Since $C_{c}^{\infty}(0,1)$ is dense in $L^{p, a}$, it follows that $T$ is the unique bounded on $L^{p, a}$ extension of the considered operator.

Let $f$ be a linear combination of $\psi_{n}^{\mu}$ 's, say $f=\sum_{1}^{N} a_{n} \psi_{n}^{\mu}$ (the assumption $-p(\mu+1 / 2)-1<a$ ensures that the linear span of $\left\{\psi_{n}^{\mu}: n=1,2, \ldots\right\}$ is indeed a subspace of $\left.L^{p, a}\right)$. It is now clear that $\lim _{r \rightarrow 1^{-}} T_{r} f(x)=\sum_{n=1}^{N} a_{n} \psi_{n+m}^{\nu}(x)$ for every $x \in(0,1)$. On the other hand, for a sequence $0<r_{1}<r_{2}<\ldots, r_{j} \rightarrow 1^{-}$, we have $\lim _{j \rightarrow \infty} T_{r_{j}} f(x)=T f(x)$ for almost every $x \in(0,1)$. Thus

$$
T f(x)=\sum_{n=1}^{N} a_{n} \psi_{n+m}^{\nu}(x), \quad x \text {-a.e., }
$$

and the density argument of Lemma 2.3 shows that $T$ is the unique bounded on $L^{p, a}$ extension of the considered operator.

\section{ACKNOWLEDGMENT}

The authors are highly indebted to the referee for pointing out an error in an earlier version of the paper as well as for suggesting a shorter proof of Lemma 3.1. This research was carried out during the Spring of 2003 while the first author was visiting the Instytut Matematyki of the Politechnika Wrocławska. He thanks this institution for its support and hospitality.

\section{REFERENCES}

[1] J. J. Betancor and K. Stempak, Relating multipliers and transplantation for Fourier-Bessel expansions and Hankel transform, Tohoku Math. J., 53 (2001), 109-129. MR.1808644 (2002b:42035)

[2] J. E. Gilbert, Maximal theorems for some orthogonal series I, Trans. Amer. Math. Soc., 145 (1969), 495-515. MR0252941 (40:6156)

[3] J. J. Guadalupe, M. Pérez, F. J. Ruiz and J. L. Varona, Two notes on convergence and divergence a.e. of Fourier series with respect to some orthogonal systems, Proc. Amer. Math. Soc., 116 (1992), 457-464. MR.1096211 (92m:42032)

[4] N. N. Lebedev, Special functions and its applications, Dover, New York, 1972. MR 0350075 $(50: 2568)$

[5] B. Muckenhoupt, Transplantation theorems and multiplier theorems for Jacobi series, Mem. Amer. Math. Soc., 356 (1986). MR0858466 (88c:42035)

[6] B. Muckenhoupt, R. L. Wheeden and W.-S. Young, Sufficiency conditions for $L^{p}$ multipliers with general weights, Trans. Amer. Math. Soc., 300 (1987), 463-502. MR0876462 (88e:42016b)

[7] B. Muckenhoupt and E. M. Stein, Classical expansions and their relation to conjugate harmonic functions, Trans. Amer. Math. Soc., 118 (1965), 17-92. MR0199636 (33:7779)

[8] K. Stempak, On connections between Hankel, Laguerre and Jacobi transplantation, Tohoku Math. J., 54 (2002), 471-493. MR.1936265 (2003h:42045)

[9] G. N. Watson, A treatise on the theory of Bessel functions, Cambridge University Press, Cambridge, 1958. MR1349110 (96i:33010) (review of the second (1944) edition)

[10] A. Zygmund, Trigonometric series, Vols. I and II, Cambridge University Press, Cambridge, 1959. MR0107776 (21:6498)

Departamento de Matemáticas y Computación, Universidad de la Rioja, Edificio

J. L. Vives, Calle Luis de Ulloa S/n, 26004 Logroño, Spain

E-mail address: oscar.ciaurri@dmc.unirioja.es

Instytut Matematyki i Informatyki, Politechnika WrocŁawska, Wyb. Wyspiańskiego 27, 50-370 WrocŁaw, Poland

E-mail address: stempak@pwr.wroc.pl 